Cite as: Hallgató, E., Győri-Dani, D., Pekár, J., Janacsek, K., \& Nemeth, D. (2013). The differential consolidation of perceptual and motor learning in skill acquisition. Cortex, 49(4), 1073-1081.

\title{
The differential consolidation of perceptual and motor learning in skill
} acquisition

Emese Hallgató ${ }^{1}$, Dóra Győri-Dani ${ }^{1}$, Judit Pekár ${ }^{1}$, Karolina Janacsek ${ }^{1}$, Nemeth Dezso ${ }^{1,2} *$

${ }^{1}$ Institute of Psychology, University of Szeged, Szeged, Hungary

${ }^{2}$ Center for the Brain Basis of Cognition, Georgetown University, Washington DC, USA

All authors contributed equally to this work

Running title: Perceptual-motor consolidation

*Corresponding author: Dezso Nemeth, Ph.D., Institute of Psychology, University of Szeged, Egyetem u. 2., 6722, Szeged, HUNGARY, Email: nemethd@edpsy.u-szeged.hu, Phone: +36 62544 692, +36 62544 509, Fax: +36 62544509 


\section{Abstract}

Implicit skill learning is an unconscious way of learning which underlies not only motor but also cognitive and social skills. This form of learning is based on both motor and perceptual information. Although many studies have investigated the perceptual and motor components of "online" skill learning, the effect of consolidation on perceptual and motor characteristics of skill learning has not been studied to our knowledge. In our research we used a sequence learning task to determine if consolidation had the same or different effect on the perceptual and the motor components of skill acquisition. We introduced a 12-hour (including or not including sleep) and a 24-hour (diurnal control) delay between the learning and the testing phase with AM-PM, PM-AM, AM-AM and PM-PM groups, in order to examine whether the offline period had differential effects on perceptual and motor learning. Although both perceptual and motor learning were significant in the testing phase, results showed that motor knowledge transfers more effectively than perceptual knowledge during the offline period, irrespective of whether sleep occurred or not and whether there was a 12- or 24-hour delay period between the learning and the testing phase. These results have important implications for the debate concerning perceptual/motor learning and the role of sleep in skill acquisition.

Keywords: consolidation, implicit skill learning, offline learning, perceptual-motor learning, sleep 


\section{Introduction}

Implicit skill learning occurs when information is acquired from an environment of complex stimuli without conscious access either to what was learned or to the fact that learning occurred (Reber, 1993). In everyday life, this learning mechanism is crucial for adapting to the environment and evaluating events. Implicit skill learning underlies not only motor but cognitive and social skills as well, it is therefore an important aspect of life from infancy to old age. Skill learning does not occur only during practice, in the so-called online periods, but also between practice periods, during the so-called offline periods. The process that occurs during the offline periods is referred to as consolidation which means stabilization of a memory trace after the initial acquisition. This process can result in increased resistance to interference or even improvement in performance following an offline period (Krakauer and Shadmehr, 2006; Nemeth et al., 2010b; Robertson, 2009; Song, 2009). Most models of skill learning (Dennis and Cabeza, 2011; Doyon et al., 2009a; Hikosaka et al., 1999; Hikosaka et al., 2002; Keele et al., 2003; Kincses et al., 2008) highlight the role of the basal ganglia and the cerebellum. One of the main debates in the field of skill learning is whether we rely on "our hands" or on "our eyes" (Deroost and Soetens, 2006; Keele et al., 2003; Mayr, 1996; Nemeth et al., 2009; Song et al., 2008; Ziessler and Nattkemper, 2001)? The goal of the present study is to determine if an offline period modifies the contribution of motor and perceptual components to implicit sequence learning. This issue is of particular interest because it deals with the question of whether sequence learning and consolidation are mediated by perceptual or by motor brain networks primarily (Deroost and Soetens, 2006; Goschke, 1998). 
One of the most popular implicit learning tasks is the Serial Reaction Time (SRT) Task (Nissen and Bullemer, 1987) and its modification, the Alternating Serial Reaction Time (ASRT) Task (Howard and Howard, 1997; Nemeth et al., 2010b). In the original version a stimulus appears at one of four possible locations on the screen, and subjects have to press the button corresponding to that location. Unbeknownst to them, the sequence of subsequent locations (and correspondingly, the sequence of the responses) follows a predetermined order. Without becoming aware of the sequence, subjects learn the regularity - and as they learn, they produce faster and more accurate responses. When the sequence is changed to a random series of stimuli, subjects become slower and less accurate in responding. In this paradigm, however, it is not clear what exactly the subjects learn: they might learn the sequence of the stimuli (perceptual learning), the sequence of their own eye movements (oculomotor learning), the sequence of response locations (response-based learning) or the sequence of given fingers' movements (effector-based learning) (Cohen et al., 1990; Remillard, 2003; Willingham, 1999).

In a SRT study Willingham (1999) used two conditions to examine the perceptual and the motor factors of learning. In one condition the stimulus-response mapping was changed in the transfer (test) phase that followed the learning phase, so that half of the subjects had to press the same sequence of keys as in the learning phase but saw new stimuli, whereas the other half had to press a different sequence of keys as in the learning phase but saw the same stimuli as before. Willingham (1999) found that transfer was shown only when the motor sequence was kept constant, but not when the perceptual sequence was constant. In a previous study, Nemeth et al. (Nemeth et al., 2009) compared the magnitude of perceptual and motor implicit sequence learning using a modification of the ASRT-task in a similar design. This 
task (ASRT-Race) contains second-order probabilistic sequences compared to classical SRT tasks that use deterministic sequences. ASRT-Race allows measuring "pure" sequence learning separate from general skill improvements, where sequence learning is reflected in the difference between the reaction times to more predictable events as opposed to less predictable ones. In addition, this task eliminates the possibility of oculomotor learning as stimuli always appear in the same central position on the screen. In contrast to Willingham's findings, Nemeth et al. (2009) demonstrated that not only motor, but perceptual learning of second-order probabilistic sequences is possible. Furthermore, Nemeth et al. (2009) showed that the two types of learning do not differ significantly in magnitude. The weakness of the above mentioned perceptual-motor studies (Deroost and Soetens, 2006; Mayr, 1996; Nemeth et al., 2009; Remillard, 2003, 2009; Song et al., 2008; Willingham, 1999) is that experiments were conducted in one session. Using only one session for measuring skill learning relates to short-term performance changes in behavior and not to more permanent changes associated with learning. Consequently, it is important to address the question of the role of offline periods in perceptual and motor skill learning.

Recent reviews indicate that whether offline improvements occur at all, and whether they are sleep-dependent, varies with factors such as awareness, the formation of contextual associations and type of information to be learned (Debas et al., 2010; Doyon et al., 2009b; Nemeth et al., 2010b; Robertson, 2009; Robertson et al., 2004; Siengsukon and Boyd, 2008; Song, 2009; Song et al., 2007). For example, Robertson (2009) argues that the consolidation of explicit (goal-directed) and implicit (movement-based) learning is differentially affected by sleep and wakefulness. In implicit learning when there is no declarative knowledge about the task, consolidation may occur during both wakefulness and sleep. In line with the predictions of this 
theory, recent SRT studies found similar consolidation of implicit skills during both sleep and wakefulness (Nemeth et al., 2010b; Robertson et al., 2004; Song et al., 2007).

Although many researches have investigated the perceptual and motor components of "online skill learning", to our knowledge, the effect of consolidation on perceptual and motor characteristics of skill acquisition has not been investigated so far (Deroost and Soetens, 2006; Mayr, 1996; Nemeth et al., 2009; Remillard, 2003, 2009; Song et al., 2008). In our study we used the ASRT-Race task (Nemeth et al., 2009) to examine the possible difference in the magnitude of motor and perceptual learning after a 12-hour and a 24-hour retention period. In addition, we also aimed at exploring the role of sleep in offline consolidation of these two factors of skill learning. Therefore a 12-hour delay was administered between the Learning Phase and Transfer Phase of the experiment, during which participants either had a sleep (night group) or they were awake (day group). If both groups acquire the same level of skill in the Learning Phase, any difference between them in the Transfer Phase will answer the question whether the perceptual or the motor component stabilizes more effectively during the offline period. In order to avoid a time-of-day effect we also administered a 24-hour delay condition.

\section{Methods}

\subsection{Participants}

There were 102 individuals (students attending the University of Szeged) in the experiment (mean age $=22.34, \mathrm{SD}=3.82 ; 44$ males, 58 females). None of them suffered from any developmental, psychiatric or neurological disorders. Participants were randomly assigned to the perceptual group or to the motor group. The perceptual 
and motor groups were further divided by the length of delay (12- or 24-hour delay) and by the daytime (morning first, AM-PM/AM-AM and evening first, PM-AM/PMPM) (see Table 1). The eight experimental groups did not differ in their sleep quality $(F(7,89)=0,98, p=0.45)$ measured by the Pittsburgh Sleep Quality Index (Buysse and Reynolds, 1989) (Due to data collection scheduling problems 5 out of 102 participants failed to administer this test). All individuals provided signed informed consent, and received no financial compensation for their participation.

Table 1. General data of participants

\begin{tabular}{lllcc}
\hline Condition & Delay & Daytime & Mean Age (SD) & N (Male/Female) \\
\hline \multirow{4}{*}{ Perceptual } & \multirow{2}{*}{ 12-hour } & Morning first (AM-PM) & $20.82(1.60)$ & $11(4 / 7)$ \\
& & Evening first (PM-AM) & $22.75(3.74)$ & $11(7 / 4)$ \\
& \multirow{2}{*}{ 24-hour } & Morning first (AM-AM) & $23.72(5.66)$ & $14(4 / 10)$ \\
& \multirow{3}{*}{ Motor } & Evening first (PM-PM) & $21.63(2.16)$ & $14(6 / 8)$ \\
& \multirow{2}{*}{ 24-hour } & Morning first (AM-PM) & $22.62(3.98)$ & $12(8 / 4)$ \\
& & Evening first (PM-AM) & $22.00(1.84)$ & $11(4 / 7)$ \\
& & Morning first (AM-AM) & $20.40(2.01)$ & $12(3 / 9)$ \\
& & Evening first (PM-PM) & $23.93(5.48)$ & $17(8 / 9)$ \\
\hline
\end{tabular}

\subsection{Procedure}

All participants completed two sessions: a Learning Phase (Session 1) and a Transfer Phase (Session 2), separated by a 12-hour or a 24-hour delay (Figure 1). For the night groups, Session 1 was in the evening (between 7 and 9 pm), and Session 2 was in the morning (between 7 and 9 am), with the opposite arrangement for the day groups. Thus, the offline period of the night group contained sleep, while the day group was awake during the offline period (Figure 1). Although previous studies with similar tasks and experimental designs showed no time of day effect either on general reaction times or on learning measures (Nemeth et al., 2010b; Press et al., 2005; 
Robertson et al., 2004; Song et al., 2007), we administered a 24-hour delay condition.

For the morning diurnal groups, both Session 1 and Session 2 were in the morning (between 7 and 9 am) and for the evening diurnal groups, both Session 1 and Session 2 took place in the evening (between 7 and $9 \mathrm{pm}$ ).

a)

I. LEARNING PHASE

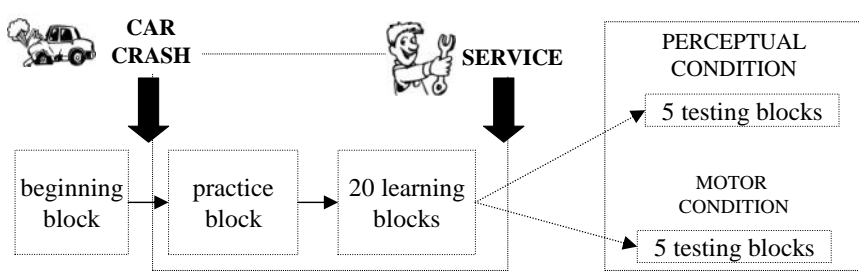

b)

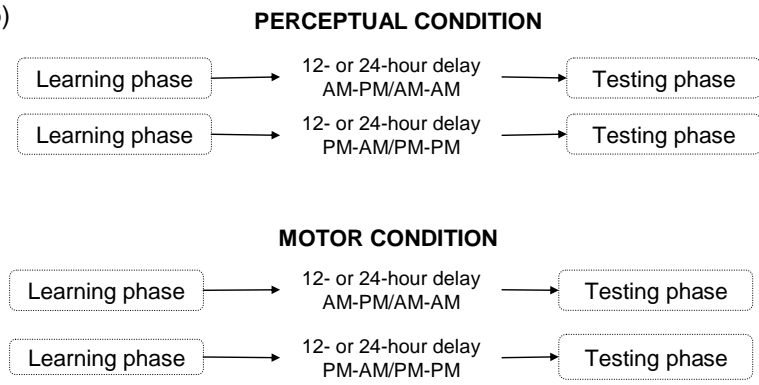

c)

I. LEARNING PHASE

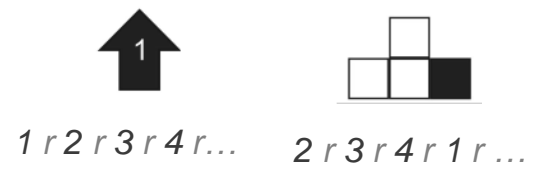

II. TESTING PHASE

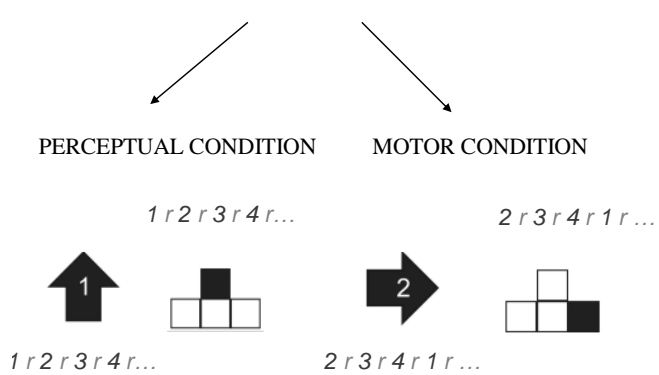

Figure 1. Design of the experiment. (a) All participants completed the ASRT-Race sequence learning task in two sessions. There were 20 learning blocks in Session 1 and 5 testing blocks in Session 2. (b) The two sessions were separated by either a 12-hour delay (in which participants had or had not slept) or a 24-hour delay. (c) In Session 2, half of the subjects were administered a new sequence which they had not seen before, but whose motor information corresponded to that of they had practiced in Session 1 (motor condition), while the other half of subjects were administered to the same perceptual information as in Session 1, but the pattern of motor responses changed due to the lack of mental rotation (perceptual condition).

\subsection{Task}

A modified version of the original ASRT (Howard and Howard, 1997) was used, the so-called ASRT-Race (Nemeth et al., 2009) in which the participants were 
instructed to drive an imaginary car on the road, as fast and as accurately as possible. The stimuli were the left, right, up and down arrows $(5 \mathrm{~cm}$ long and $3 \mathrm{~cm}$ wide) appearing in the center of the screen, and representing the direction the car had to be steered. For example, when the subjects saw the right arrow, they had to press the right button on the keyboard to make a right turn with the car. All participants pressed the keys with their right hand.

Session 1 consisted of 22 blocks, starting with a block containing 85 random presses (excluded from data analysis), after which the individuals were told that there was a car crash and the steering wheel failed. Due to the defective steering wheel they had to mentally rotate the arrows appearing on the screen by $90^{\circ}$, and press the keyboard button designated to the rotated arrow, in order to maneuver the car in the right direction (Figure 1a). For instance, if they saw the up arrow on the screen they had to press the right arrow on the keyboard, if they saw the right arrow they had to press the down arrow button, and so on (Figure 1c). After the change in the instruction, there were 21 blocks, starting with 1 random block, in which participants could practice the new rules regarding the mental rotation, followed by 20 learning blocks (Learning phase). Each of the 20 learning blocks contained 85 key presses. The initial 5 stimuli were random (warm-up; excluded from data analysis), then an eightelement sequence alternated 10 times. Since the ASRT task is based on a non-adjacent sequence, random and sequence elements alternate one after the other. For example 2-R $-3-\mathrm{R}-1-\mathrm{R}-4-\mathrm{R}$, where $\mathrm{R}$ represents random trials and the numbers represent the sequence-specific elements, implicating the arrows' direction (1-up, 2-right, 3-down, 4-left). The stimulus remained on the screen until the participant pressed the correct button. The next arrow appeared following a 120-ms delay (response to stimulus interval) after the subject's correct response. These parameters are consistent with the 
original task presented by Howard and Howard (1997). During this delay, a fixation cross was displayed on the screen. Participants were told to respond as fast and as accurately as they could.

Session 2 (Transfer Phase) took place either after a 12-hour or a 24-hour delay. The Transfer Phase consisted of 5 blocks. In this session participants were told that the car had been taken to a mechanic, and the steering wheel had been fixed, so they could use the answer keys corresponding to the arrows appearing on the screen (right button for right arrow, down button for down arrow, etc.). Half of the subjects participated in the motor condition, while the other half was assigned to the perceptual condition. Subjects in the motor condition were administered a new sequence which they had not seen before, but whose motor information corresponded to the one of they had practiced in Session 1, while subjects in the perceptual condition were administered to the same perceptual information as in Session 1, but the pattern of motor responses changed due to the lack of mental rotation (Figure 1c). Thus, while in Session 1 all subjects performed the same task, in Session 2 they were divided into two groups (perceptual vs. motor). The difference between the two groups allowed us to separate the motor and the perceptual information of the sequence previously learnt by the subjects. In this way we could determine whether the perceptual and the motor component had the same or different effects on learning. All the stimuli were displayed in the center of the screen in order to exclude the possible oculomotor aspect of learning. After Session 2, we administered a short questionnaire regarding the participants' possible explicit knowledge about the task (Song et al., 2007). In keeping with other probabilistic SRT studies (Jiménez et al., 2006; Nemeth et al., 2010b; Song et al., 2007), none of them reported having noticed the sequences. 


\subsection{Data analysis}

Since the core structure of the tasks was the same as in the original ASRT, we followed the same procedures in our analysis (Howard and Howard, 1997; Nemeth et al., 2010b). As there is a fixed sequence in the ASRT-Race with alternating random elements (also known as non-adjacent sequence) (Remillard, 2008), for example 2-R$3-\mathrm{R}-1-\mathrm{R}-4-\mathrm{R}$, some triplets or runs of three events occur more frequently than others. For instance, following the illustration above, triplets such as $2 \_3,3 \_1,1 \_4$, 4_2 (where " " indicates the middle element of the triplet) can occur more often, because the third element (bold numbers) could be derived from the sequence, or could also be a random element. In contrast, triplets such as $4 \_\mathbf{1}, 4 \_\mathbf{4}$ would occur infrequently, because in this case the third element could only be random. Following previous studies, we refer to the former as high-frequency triplets and the latter as low-frequency triplets. Because of this difference in frequencies of certain triplets, after observing two stimuli, a certain third stimulus can be expected with $62,5 \%$ probability (for example, 223 is five times more probable than 221 or 222 or 224). In our analysis, we determined for every stimulus if it was the more probable or the less probable continuation for the previous trials (see Figure 2). Participants are faster at the probable stimuli than at the less probable ones, revealing sequence learning in the ASRT paradigm (Howard et al., 2004; Song et al., 2007). 
a) SLE Learning Phase

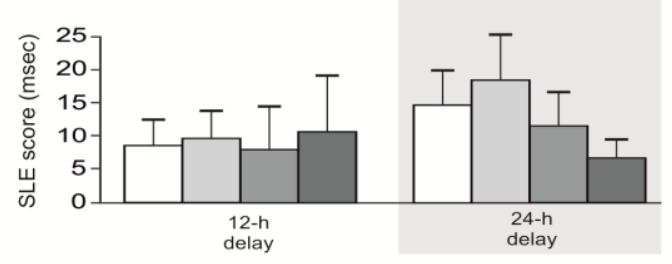

) SLE Transfer Phase

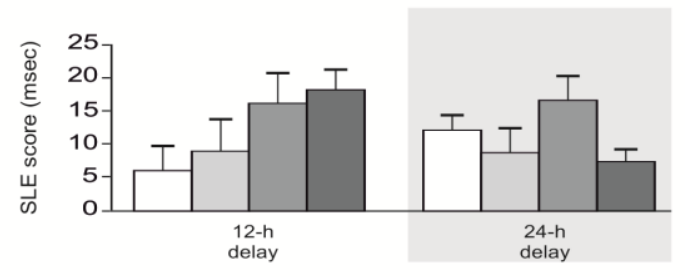

c) SLE difference

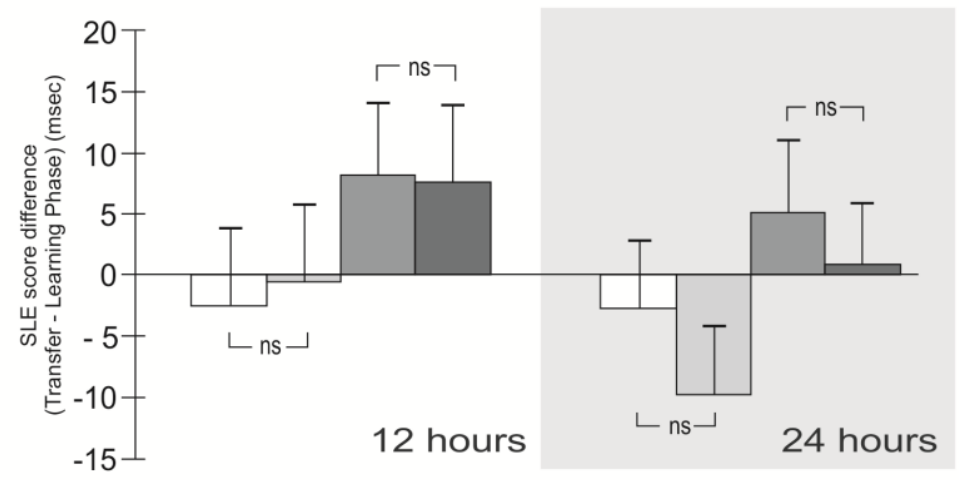

PERCEPTUAL group, morning first PERCEPTUAL group, evening first MOTOR group, morning first MOTOR group, evening first

Figure 2. In a typical ASRT sequence, there are more frequent (high frequency) triplets and less frequent (low frequency) triplets. In other words, if we know what were the last two elements of the sequence (in this case $2-3-?$ ), there is a $62.5 \%$ probability of a certain element as continuation, and only $12.5 \%$ probability of all of the other elements.

Similar to prior investigations, two kinds of low-frequency triplets were excluded from the analysis; trills (e.g. 121, 434) and repetitions (e.g. 111, 222). These triplets are low frequency for all individuals, and people often show pre-existing response tendencies to them. By eliminating these triplets, we can assure that any high versus low frequency differences are due to learning, and not pre-existing tendencies (Howard et al., 2004; Nemeth et al., 2009; Nemeth et al., 2010b).

Since the accuracy of the participants was very high (average over $94.92 \%$ in all groups, in all phases), our analysis focused on RT data. For statistical analysis, median RTs were calculated for correct responses only, for each subject for every five blocks, both for the low-frequency and high-frequency elements. 
To define the index for Sequence Learning Effect (SLE) (Nemeth and Janacsek, 2011; Nemeth et al., 2010a; Song et al., 2007; Song et al., 2009), we calculated the RT difference between the low and high frequency triplets separately in the Learning Phase (Session 1) and in the Transfer Phase (Session 2) for every five blocks. As we subtracted mean RT of high frequency from low frequency triplets, SLE was a positive number only if sequence learning occurred, a larger value indicating a stronger effect.

\section{Results}

\subsection{Learning in Session 1}

To be able to investigate the effect of transfer after 12- and 24-hour delay, the learning in Session 1 must be similar in the groups. From this point of view, the end of Session 1 is crucial (Nemeth and Janacsek, 2011; Nemeth et al., 2010b; Press et al., 2005; Song et al., 2007). Therefore, we analyzed the SLE of the last five blocks of the Learning Phase for every group. Univariate ANOVA was conducted with CONDITION (perceptual vs. motor), DAYTIME (morning first vs. evening first groups) and DELAY (12- and 24-hour) as between-subject factors. ANOVA revealed significant sequence learning $(F(1,94)=32.31, \mathrm{p}<0.001)$ which is inferred from the test whether the overall mean is different from zero (Mean SLE=11.16 ms). There were no other significant main effects or interactions involving CONDITION, DAYTIME and DELAY (all p>0.32), thus these between-subject factors had no significant effect on sequence learning.

\subsection{Transfer of Sequence Learning Effect from Session 1 to Session 2}


To determine whether the performance in Session 2 declined, improved, or was constant in relationship to the end of Session 1, we subtracted the SLE score of the last five blocks of the Learning Phase from the SLE score of the Transfer Phase (Transfer-SLE). As the groups were similar in SLE at the end of Session 1 (Learning Phase), any difference among groups in Transfer-SLE could be attributed to the differential effects of consolidation. We conducted a univariate ANOVA for this Transfer-SLE score with CONDITION (perceptual vs. motor), DAYTIME (morning first vs. evening first groups) and DELAY (12- and 24-hour) as between-subject factors. ANOVA revealed a main effect of CONDITION $(F(1,94)=4.92, p=0.029)$, the motor group showing larger SLE than the perceptual group (Figure 3). ANOVA showed no significant main effect or interaction with DAYTIME (all p>0.45), suggesting that the AM-PM, PM-AM, AM-AM and PM-PM groups did not differ in their SLE. In addition, main effect and interactions with DELAY were not significant either (all p>0.25), suggesting that 12- and 24-hour delay groups performed at a similar level.

Thus, the only significant effect in the ANOVA was the main effect of CONDITION, suggesting differential consolidation of perceptual and motor groups with better consolidation for the motor group, irrespective of the delay or daytime. Despite this difference in consolidation, SLE in Session 2 was significantly different from zero for both the perceptual and motor groups (one-sample t-tests for SLE scores: $\mathrm{t}(49)=5.25, \mathrm{p}<0.001$ and $\mathrm{t}(51)=8.72, \mathrm{p}<0.001$ respectively). Thus, in spite of the weaker consolidation in the perceptual group, they still showed significant SLE in the Transfer Phase (Session 2). For detailed descriptive statistics see Appendix 1. 
a) SLE Learning Phase

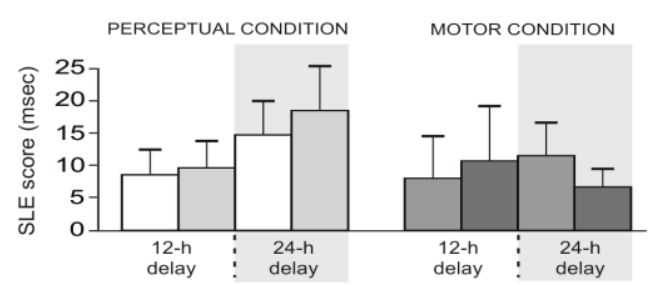

b) SLE Transfer Phase

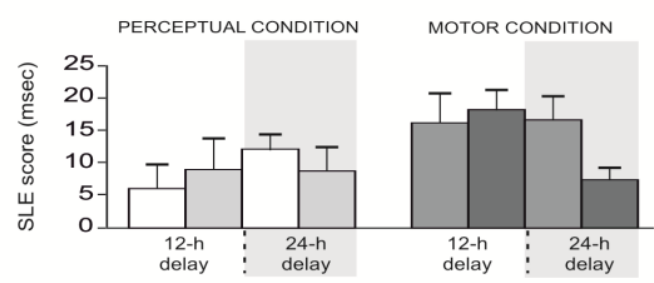

c) SLE difference

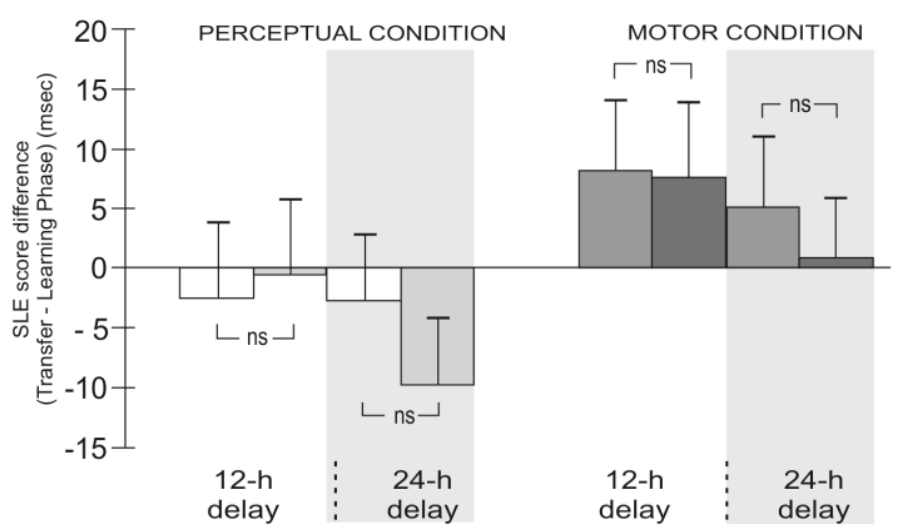

PERCEPTUAL group, morning first PERCEPTUAL group, evening first MOTOR group, morning first MOTOR group, evening first

Figure 3. A) SLE score (Sequence Learning Effect) of each experimental group in the last 5 blocks of the Learning Phase. B) SLE score of each experimental group in the Transfer Phase (Session 2). C) Difference between SLE scores of the 5 blocks of Transfer phase and the last 5 blocks of Learning phase (Transfer SLE score). The perceptual groups showed weaker transfer effect than the motor groups both after 12 and 24 hours. Error bars indicate Standard Error of Mean.

\subsection{Transfer or new motor learning in Perceptual Group?}

In order to find out whether the significant learning effect in Transfer Phase (Session 2) is due to new motor learning in the perceptual group we investigated the learning effect at the beginning of the Learning Phase (Session 1 - the first two sequence blocks) and learning effect in the Transfer Phase (Session 2 - Block 1-2) separately. We calculated SLE scores for the first 2 blocks of Session 1 and Session 2. We submitted these scores to a one-sample t-test separately for Session 1 and Session 2. If we can show a significant learning effect in Session 1 - Block 1-2, the learning is very fast; and the results in Session 2 can be due to new motor learning. However, we 
found no significant learning effect in Session 1 - Block 1-2 in the perceptual group (one-sample t-test for SLE-score: $\mathrm{t}(49)=-1.069, \mathrm{p}=0.291$, Mean SLE=-9.27). In contrast we found a significant learning effect in Session 2 - Block 1-2 (one-sample ttest for SLE-score: $\mathrm{t}(49)=3.523, \mathrm{p}=0.001$, Mean $\mathrm{SLE}=8.33$ ). Hence it is likely that the learning effect in Session 2 (Transfer Phase) is attributable to preserved perceptual learning rather than to new motor learning. We found the same pattern in the motor condition (one-sample t-test for SLE-score in Session 1 - Block 1-2: $t(51)=0.3$, $\mathrm{p}=0.765$, Mean SLE=3.89; Session $2-$ Block 1-2: $\mathrm{t}(51)=5.087, \mathrm{p}<0.001$, Mean SLE=14.77 ). For detailed descriptive statistics see Appendix 2.

\section{Discussion}

Our study investigated the role of 12-hour and 24-hour delay on perceptual and motor components of implicit skill learning, while eliminating oculomotor learning. In this way we connect two debates together: 1) one on the relative importance of perceptual and motor learning 2) the other on the effect of sleep on skill acquisition. We used the same method as Nemeth et al.'s study (2009), except that in our research there was a 12-hour (during which participants either had sleep or they were awake) or a 24-hour (diurnal) offline period between the Learning and the Transfer Phase. We found significant sequence learning in the Learning Phase. After the 12-hour and the 24-hour offline period we found significant learning effect in both the perceptual and the motor conditions, however transfer in the motor condition was more effective compared to the perceptual condition. We did not find any sleep-effect on sequence learning in either condition.

The weaker consolidation of perceptual learning is in agreement with the results of Deroost \& Soetens (2006) and Willingham (1999), who found no evidence 
of perceptual learning except for specific conditions. According to previous studies, perceptual learning only takes place when the structure of the sequence is simple, but in case of deterministic sequences with second-order dependencies and probabilistic sequences with first-order dependencies perceptual learning is not or only weakly present (Deroost and Soetens, 2006; Mayr, 1996; Remillard, 2003). Also, previous studies found perceptual learning in explicit conditions (Russeler and Rosler, 2000), and when a motor sequence was learnt concurrently (Mayr, 1996). In our study participants had no conscious awareness at all of the structure of the sequence, as the ASRT task uses probabilistic sequences with second-order dependencies. The only condition that met Deroost's (2006) criteria is that in the Learning Phase participants learnt the perceptual and motor components concurrently. Compared to Nemeth et al. (2009) who found similar magnitudes of perceptual and motor learning immediately after the Learning Phase, we found a weaker perceptual learning effect in the Transfer phase both after a 12-hour and a 24-hour delay. Because the only difference was the delay duration, we can suppose that the differences between the results of the two studies can be related to the consolidation period. Thus, this one criterion (i.e. participants in the Learning Phase learnt the perceptual and motor components concurrently) can be enough for finding significant perceptual learning immediately after the Learning Phase (Meier and Cock, 2010; Nemeth et al., 2009; Weiermann et al., 2010), however, it might result in weaker consolidation after the delay period. To put the puzzle together, based on the present study we can propose that the consolidation period has a differential effect on motor and perceptual components of learning, such that in the Transfer Phase the motor learning effect is larger than the perceptual one. 
Song et al. (2008), Nemeth et al. (2009) and the present study are similar in the nature of the sequence structure and the implicitness of the task. Furthermore, the present study and the study of Nemeth et al. (2009) also eliminated the possibility of oculomotor learning. Because we focused only on the perceptual and motor learning while controlling for the oculomotor learning, the role of response-based learning and effector-based learning remained unclear (Cohen et al., 1990; Remillard, 2003; Willingham, 1999); therefore the exact nature of the underlying mechanism still needs to be investigated.

In addition to the question of perceptual and motor components of learning, our study has relevance for the sleep debate in skill consolidation (Debas et al., 2010; Doyon et al., 2009b; Gerván and Kovács, 2007; Karni, 1994; Robertson, 2009; Song, 2009; Stickgold and Walker, 2005; Walker et al., 2002). As pointed out by Robertson (2009) and supported by Song et al. (2007) and Nemeth et al. (2010b), we found that sleep does not support sequence learning. In addition, sleep has no different role in the consolidation of motor and perceptual factors of implicit sequence learning. A plausible explanation can be that in the probabilistic sequence learning task used in this study, besides primary sensory and motor brain regions, sub-cortical structures and cerebellum are more involved (Doyon, 2008; Hikosaka et al., 1999; Hikosaka et al., 2002), opposed to the more basic finger tapping tasks where sleep-dependent improvement was usually found (Walker et al., 2002).

To conclude, despite the 12-hour or the 24-hour offline period we found a significant perceptual and motor learning effect in the Transfer Phase, however the transfer of motor knowledge was more robust, irrespective of whether sleep occurred in the consolidation period or not. These results have important implications for the perceptual/motor and also for the sleep debate in skill learning in the following ways: 
1) Previous experiments in this field included only one session which can reveal short-term performance changes in behavior. Consequently, it is important to use more sessions with many hours (even a day) delay between sessions for measuring permanent changes in neural plasticity. 2) Sleep has no contribution to this type of learning. However, further investigations need to explore more deeply conditions (including nature of sequence, awareness, perceptual/motor learning) in which sleep has a significant role in skill learning. 3) The retention period itself (regardless of sleep) has a modifying effect on the consolidation of perceptual/motor knowledge and the underlying brain networks.

\section{Acknowledgements}

Thanks to our mentors: Darlene V. Howard and James H. Howard, Jr. from Georgetown University. This research was supported by Bolyai Scholarship Program (D. N.) and OTKA K 82068. Thanks to Ágnes Szokolszky and Szabolcs Kéri helping us with the final version of the manuscript.

\section{References}

Buysse D and Reynolds C. The Pittsburgh Sleep Quality Index: a new instrument for psychiatric practice and research. Psychiatry Research, 28 (2): 193-213, 1989.

Cohen A, Ivry RI, and Keele SW. Attention and structure in sequence learning. Journal of Experimental Psychology: Learning, Memory, and Cognition, 16 (1): $17-$ 30, 1990.

Debas K, Carrier J, Orban P, Barakat M, Lungu O, Vandewalle G, Tahar AH, Bellec P, Karni A, Ungerleider LG, Benali H, and Doyon J. Brain plasticity related to the consolidation of motor sequence learning and motor adaptation. Proceedings of the 
National Academy of Sciences of the United States of America, 107 (41):1783917844, 2010.

Dennis NA and Cabeza R. Age-related dedifferentiation of learning systems: an fMRI study of implicit and explicit learning. Neurobiology of Aging, 32 (12): 2318.e17-30, 2011.

Deroost N and Soetens E. Perceptual or motor learning in SRT tasks with complex sequence structures. Psychological Research, 70 (2): 88-102, 2006.

Doyon J. Motor sequence learning and movement disorders. Current Opinion in Neurology, 21 (4): 478-483, 2008.

Doyon J, Bellec P, Amsel R, Penhune V, Monchi O, Carrier J, Lehericy S, and Benali H. Contributions of the basal ganglia and functionally related brain structures to motor learning. Behavioural Brain Research, 199 (1): 61-75, 2009a.

Doyon J, Korman M, Morin A, Dostie V, Hadj Tahar A, Benali H, Karni A, Ungerleider LG, and Carrier J. Contribution of night and day sleep vs. simple passage of time to the consolidation of motor sequence and visuomotor adaptation learning. Experimental Brain Research, 195 (1): 15-26, $2009 \mathrm{~b}$.

Gerván P and Kovács I. Sleep dependent learning in contour integration. Journal of Vision, 7 (9): 48, 2007.

Goschke T. Implicit learning of perceptual and motor sequences: Evidence for independent learning systems. in: Stadler, M.A., Frensch, P.A. (Eds.), Handbook of implicit learning. SAGE Publications: London, 1998. pp. 401-444.

Hikosaka O, Nakahara H, Rand MK, Sakai K, Lu X, Nakamura K, Miyachi S, and Doya K. Parallel neural networks for learning sequential procedures. Trends in Neurosciences, 22 (10): 464-471, 1999. 
Hikosaka O, Nakamura K, Sakai K, and Nakahara H. Central mechanisms of motor skill learning. Current Opinion in Neurobiology, 12 (2): 217-222, 2002.

Howard DV, Howard Jr JH, Japikse K, DiYanni C, Thompson A, and Somberg R. Implicit sequence learning: effects of level of structure, adult age, and extended practice. Psychology and Aging, 19 (1): 79-92, 2004.

Howard Jr JH and Howard DV. Age differences in implicit learning of higher-order dependencies in serial patterns. Psychology and Aging, 12 (4): 634-656, 1997.

Jiménez L, Vaquero J, and Lupiá ez J. Qualitative differences between implicit and explicit sequence learning. Journal of Experimental Psychology-Learning Memory and Cognition, 32 (3): 475-489, 2006.

Karni A. Dependence on Rem-sleep of overnight improvement of a perceptual skill. Science, 265 (5172): 679-682, 1994.

Keele SW, Ivry R, Mayr U, Hazeltine E, and Heuer H. The cognitive and neural architecture of sequence representation. Psychological Review, 110 (2): 316-339, 2003.

Kincses T, Johansen-Berg H, Tomassini V, Bosnell R, Matthews P, and Beckmann C. Model-free characterization of brain functional networks for motor sequence learning using fMRI. Neuroimage, 39 (4): 1950-1958, 2008.

Krakauer JW and Shadmehr R. Consolidation of motor memory. Trends in Neurosciences, 29 (1): 58-64, 2006.

Mayr U. Spatial attention and implicit sequence learning: Evidence for independent learning of spatial and nonspatial sequences. Journal of Experimental Psychology, 22 (2): 350-364, 1996.

Meier B and Cock J. Are correlated streams of information necessary for implicit sequence learning? Acta psychologica, 133 (1): 17-27, 2010. 
Nemeth D, Hallgato E, Janacsek K, Sandor T, and Londe Z. Perceptual and motor factors of implicit skill learning. Neuroreport, 20 (18): 1654-1658, 2009.

Nemeth D and Janacsek K. The dynamics of implicit skill consolidation in young and elderly adults. Journal of Gerontology Psychological Science, 66 (1): 15-22, 2011.

Nemeth D, Janacsek K, Balogh V, Londe Z, Mingesz R, Fazekas M, Jambori S, Danyi I, and Vetro A. Learning in Autism: Implicitly Superb. PLoS ONE, 5 (7): e11731, 2010a.

Nemeth D, Janacsek K, Londe Z, Ullman MT, Howard D, and Howard J. Sleep has no critical role in implicit motor sequence learning in young and old adults. Experimental Brain Research, 201 (2): 351-358, 2010 b.

Nissen MJ and Bullemer P. Attentional requirements of learning: Evidence from performance measures. Cognitive Psychology, 19: 1-32, 1987.

Press DZ, Casement MD, Pascual-Leone A, and Robertson EM. The time course of off-line motor sequence learning. Brain Research. Cognitive Brain Research, 25 (1): 375-378, 2005.

Reber AR. Implicit learning and tacit knowledge: An essay on the cognitive unconscious. Oxford University Press: New York, 1993.

Remillard G. Pure perceptual-based sequence learning. Journal of Experimental Psychology: Learning, Memory, and Cognition, 29 (4): 581-597, 2003.

Remillard G. Implicit learning of second-, third-, and fourth-order adjacent and nonadjacent sequential dependencies. The Quarterly Journal of Experimental Psychology, 61 (3): 400-424, 2008.

Remillard G. Pure perceptual-based sequence learning: A role for visuospatial attention. Journal of Experimental Psychology: Learning, Memory, and Cognition, 35 (2): 528, 2009. 
Robertson EM. From creation to consolidation: A novel framework for memory processing. PLoS Biology, 7 (1): e1000019, 2009.

Robertson EM, Pascual-Leone A, and Press DZ. Awareness modifies the skilllearning benefits of sleep. Current Biology, 14 (3): 208-212, 2004.

Russeler J and Rosler F. Implicit and explicit learning of event sequences: Evidence for distinct coding of perceptual and motor representations. Acta Psychologica, 104 (1): 45-67, 2000.

Siengsukon CF and Boyd LA. Sleep enhances implicit motor skill learning in individuals poststroke. Topics in Stroke Rehabilitation, 15 (1): 1, 2008.

Song S. Consciousness and the consolidation of motor learning. Behavioural Brain Research, 196 (2): 180-186, 2009.

Song S, Howard Jr JH, and Howard DV. Sleep does not benefit probabilistic motor sequence learning. Journal of Neuroscience, 27 (46): 12475-12483, 2007.

Song S, Howard Jr JH, and Howard DV. Perceptual sequence learning in a serial reaction time task. Experimental Brain Research, 189 (2): 145-158, 2008.

Song S, Marks B, Howard Jr JH, and Howard DV. Evidence for parallel explicit and implicit sequence learning systems in older adults. Behavioural Brain Research, 196 (2): 328, 2009.

Stickgold R and Walker MP. Sleep and memory: the ongoing debate. Sleep, 28 (10): $1225-1227,2005$.

Walker MP, Brakefield T, Morgan A, Hobson JA, and Stickgold R. Practice with sleep makes perfect: sleep-dependent motor skill learning. Neuron, 35 (1): 205-211, 2002.

Weiermann B, Cock J, and Meier B. What matters in implicit task sequence learning: perceptual stimulus features, task sets, or correlated streams of information? Journal 
of experimental psychology: learning, memory, and cognition, 36 (6): 1492-1509, 2010.

Willingham DB. Implicit motor sequence learning is not purely perceptual. Memory and Cognition, 27 (3): 561-572, 1999.

Ziessler M and Nattkemper D. Learning of event sequences is based on responseeffect learning: further evidence from a serial reaction task. Journal of Experimental Psychology. Learning, Memory, and Cognition, 27 (3): 595-613, 2001.

\section{Appendices}

Appendix 1. Means and standard deviations (SD) for sequence learning effects (SLE) at the end of the Learning Phase, at the beginning of the Transfer Phase. SLE-change indicates the difference in sequence learning effects between the two sessions.

\begin{tabular}{|c|c|c|c|c|c|c|c|c|c|}
\hline \multirow[t]{2}{*}{ Condition } & \multirow[t]{2}{*}{ Delay } & \multirow[t]{2}{*}{ Daytime } & \multirow[t]{2}{*}{$\mathbf{N}$} & \multicolumn{2}{|c|}{$\begin{array}{l}\text { SLE (Learning } \\
\text { Phase) }\end{array}$} & \multicolumn{2}{|c|}{$\begin{array}{l}\text { SLE (Transfer } \\
\text { Phase) }\end{array}$} & \multicolumn{2}{|c|}{$\begin{array}{c}\text { SLE-change } \\
\text { (Transfer - } \\
\text { Learning) }\end{array}$} \\
\hline & & & & Mean & SD & Mean & SD & Mean & SD \\
\hline \multirow{5}{*}{ Perceptual } & \multirow{2}{*}{ 12-hour } & Morning-first & 11 & 8.59 & 12.57 & 6.09 & 12.06 & -2.50 & 17.45 \\
\hline & & Evening-first & 11 & 9.68 & 13.27 & 9.14 & 15.68 & -0.55 & 18.47 \\
\hline & \multirow{2}{*}{ 24-hour } & Morning-first & 14 & 14.82 & 19.39 & 12.11 & 8.70 & -2.71 & 16.75 \\
\hline & & Evening-first & 14 & 18.64 & 25.42 & 8.86 & 13.64 & -9.79 & 28.29 \\
\hline & Total & & 50 & 13.39 & 18.87 & 9.22 & 12.41 & -4.17 & 20.78 \\
\hline \multirow{5}{*}{ Motor } & \multirow{2}{*}{ 12-hour } & Morning-first & 12 & 8.13 & 22.51 & 16.29 & 15.20 & 8.17 & 19.14 \\
\hline & & Evening-first & 11 & 10.73 & 27.96 & 18.36 & 9.03 & 7.64 & 27.58 \\
\hline & \multirow{2}{*}{ 24-hour } & Morning-first & 12 & 11.63 & 18.27 & 16.67 & 12.18 & 5.04 & 24.64 \\
\hline & & Evening-first & 17 & 6.68 & 11.63 & 7.56 & 6.79 & 0.88 & 12.63 \\
\hline & Total & & 52 & 9.01 & 19.53 & 13.96 & 11.54 & 4.95 & 20.46 \\
\hline Total & & & 102 & 11.16 & 19.24 & 11.41 & 14.45 & 0.48 & 21.02 \\
\hline
\end{tabular}

Appendix 2. Means and standard deviations (SDs) for the first two sequence blocks of the Learning and Transfer Phase for perceptual and motor condition.

\begin{tabular}{llcc} 
Condition & Phase & Mean & SD \\
\hline \multirow{2}{*}{ Perceptual (N=50) } & Learning & -9.27 & 61.34 \\
& Transfer & 8.33 & 16.72 \\
\multirow{2}{*}{ Motor (N=52) } & Learning & 3.89 & 93.46 \\
& Transfer & 14.77 & 20.94
\end{tabular}

